\title{
Food Additives-Ascorbic Acid Quality Assay Test Intelligent Management
}

\author{
Hairui Zhang ${ }^{1}$, Li Zhang ${ }^{2}$, Helin Ye $^{2}$ and Guofu Zhang ${ }^{2}$ \\ 1. School of Electronie and Information Engineering, Lanzhou City University, Lanzhou 730070, China \\ 2. School of Chemistry and Environmental Engineering, Lanzhou City University, Lanzhou 730070, China
}

\begin{abstract}
In this paper, based on the analysis and test methods of national standards (GB14754-2010) and chemical analysis and test items carried out by chemical enterprises, a set of automatic processing of quality analysis test data of ascorbic acid products was developed by using access database technology and Visual Basic programming language system, and its stability was investigated. The results show that the software can manage intelligently all aspects of the quality analysis and test of ascorbic acid products, uploading timely the data and results of the analysis and inspection to the network and saving it for users, enterprises and quality management, which set up a network of information sharing platform to ensure the authenticity and reliability of measurement results, improving greatly the speed of data processing, saving valuable time, reducing production costs with good economic efficiency and social benefit. It has practical value for ascorbic acid quality analysis test data processing automatically the results of the implementation of intelligent management.
\end{abstract}

Key words: Food additive, ascorbic acid, quality analysis test, intelligent management, software development.

\section{Introduction}

In today's society, the development of the Internet has brought great convenience and efficiency to people's work and life. Informationization and intelligence have become the first choice for saving costs, saving time and improving work efficiency. Currently a large number of enterprises on ascorbic acid product quality analysis test data processing and management are still in the manual operation stage. Chemical companies ascorbic acid product quality analysis test data processing and analysis of the test report book handwritten to the laboratory staff and managers have brought a lot of inconvenience, not only Inefficient, because of carelessness and mistakes in management, data are not real and data loss, analysis of test data cannot be long-term preservation, cannot check on the network. Therefore, in order to meet the needs of chemical companies in the quality analysis and test of ascorbic acid products, computer-aided

Corresponding author: Hairui Zhang, lecturer, research fields: grid and cloud computing, software development. system software of design, development and calculation results of ascorbic acid quality analysis test, data processing, product specifications evaluation and printing of quality analysis test report book was saved to help analysis of the staff asbestos product quality analysis test data intelligent management, save data processing time, improve work efficiency, and establish a complete and strong ascorbic acid product quality analysis test information database to complete the data input, processing, analysis, storage, calling queries and other functions [1].

\section{Research Significance}

Ascorbic acid is known as vitamin C, and it's molecular formula is $\mathrm{C} 6 \mathrm{H} 8 \mathrm{O}$. It is a water-soluble vitamin present in fresh vegetables and some fruits, white or light yellow crystals or crystalline powder, odorless, pickled, melting point $190 \sim 192{ }^{\circ} \mathrm{C}$. Due to the reducibility of the enediol in the molecule, it can be quantitatively oxidized to diketone by $\mathrm{I}_{2}$. The reaction equation is $\mathrm{C}_{6} \mathrm{H}_{8} \mathrm{O}_{6}+\mathrm{I}_{2}=\mathrm{C}_{6} \mathrm{H}_{6} \mathrm{O}_{6}+2 \mathrm{HI}$; the quantitative reaction of $1 \mathrm{~mol}$ of ascorbic acid with 1 
mol of $\mathrm{I}_{2}$ has the molar mass of ascorbic acid of $176.12 \mathrm{~g} \cdot \mathrm{mol}^{-1}$. Dry pure products are stable in the air, impure products and instability in the presence of natural products, in storage and pickled, easy to destroy cooking. It is soluble in water, which is slightly soluble in ethanol, which is insoluble in ether, chloroform, benzene, petroleum ether, oils and fats [1]. Ascorbic acid is reducible highly, which is easily oxidized in the air, especially in alkaline medium even more, when measured by adding HAc solution was weakly acidic to reduce the side effects of ascorbic acid, the general choice of $\mathrm{pH} 3-4$. Ascorbic acid is widely used in food, medicine and chemistry. The direct iodometric method can measure ascorbic acid in vitamin C tablets, injections, foods, beverages, vegetables, fruits and the like [2].

Ascorbic acid production process often need to ascorbic acid product quality determination, the traditional method of determination mainly by direct iodometric method for the determination of its content, it is a business laboratory routine analysis. Direct iodometric method for the determination of ascorbic acid content is more accurate, the process is simple and reliable, the results are stable and reliable, it is a more scientific method of measurement, but this method ascorbic acid determination results of the data processing are more cumbersome. The measurement results cannot be implemented network and intelligent management, and measurement results cannot be shared data. In order to overcome these shortcomings, this paper was designed and developed a software system for the automatic processing of ascorbic acid quality analysis test results data to realize the automation and intelligence of data processing and data management. At the same time, the data were saved and the test results were analyzed. The test data and analysis results were uploaded to the background database to achieve data sharing, which has very important practical significance. The system is simple and easy to operate, friendly interface, stable operation, accurate and reliable results, and has a high value for popularization and application, which provides technical support for the construction of enterprise information and is undoubtedly an innovative achievement [3].

\section{Develop the Language and Database}

\subsection{Development Language}

Based on VB (Visual Basic) language environment, the data processing, result analysis, database management, database development technology and implementation method of the food additive ascorbic acid quality analysis test were implemented. The Visual Basic language was used as the software development language. The Visual Basic language has a GUI (graphical user interface) and a RAD (rapid application development) system that makes it easy to connect to a database using DAOs (data access objects), application programming interfaces (RDOs), program objects (ADOs), or easily ActiveX control. You can build easily quickly an application using the components provided in the Visual Basic language to create quickly Windows programs in the Visual Basic language and write enterprise-level client/server programs and powerful database applications. Therefore, Visual Basic language was used as a software development language. In addition to main features described above, the Visual Basic language has many other features that support powerful database applications using ActiveX technology, web-based development, a variety of application design wizards, and comprehensive online help functions [4].

\subsection{Database (Access Database)}

The Visual Basic (6.0) language supports directly the Access database. The VB database system is a multi-layer structure composed of a user interface, a database engine and a data warehouse. The user interfaces and functions are implemented by using controls and programming code. In order to improve the data processing power, control operations are used to achieve the operation of the data table. Because 
Access database system is mature, stable, reliable and simple, it is the preferred database system for small development system. A safe, stable and efficient operating environment and technology-based was chose in the system development environment and the choice of technology.

In the choice of development environment, the single operating environment as a supplement, network operating environment-based, with the most widely used windows as a system support platform, the entire system using $\mathrm{C} / \mathrm{S}$ mode architecture, both to ensure system security sex, reliability, but also take into account the convenience of single-operation and on-line operation and connection of fluency. The iterative development method of "design-develop-apply-modify design-develop-apply" was used in the development of the system adopts [5].

\section{Research Methods}

The $0.2 \mathrm{~g}$ ascorbic acid was weighed accurately, and was placed in $250 \mathrm{~mL}$ conical flask, and then 80 $\mathrm{mL}$ freshly boiled and cooled distilled water was added, $2 \mathrm{~mL} 20 \% \mathrm{H}_{2} \mathrm{SO}_{4}$ was added in solution with shaking, titrated immediately with $\mathrm{I}_{2}$ standard solution, near the end, $3 \mathrm{~mL} 10 \mathrm{~g} \cdot \mathrm{L}^{-1}$ starch solution was added, continue dropping $\mathrm{I}_{2}$ standard solution to a stable light blue and no leg color within $30 \mathrm{~s}$ is the end point, the volume of $I_{2}$ standard solution consumed was written down, and then the percentage of ascorbic acid was calculated. According to the country standard (GB14754-2010) the loss on drying and determination of residue content on the sample was measured and the results of a statistical were analysised.

(1) Write down the chemical reaction equation: $\mathrm{C}_{6} \mathrm{H}_{8} \mathrm{O}_{6}+\mathrm{I}_{2}=\mathrm{C}_{6} \mathrm{H}_{6} \mathrm{O}_{6}+2 \mathrm{HI}$;

(2) Find out the relationship between the amount of substance between the measured object and the standard solution: $\ln \left(\mathrm{C}_{6} \mathrm{H}_{8} \mathrm{O}_{6}\right) \sim \ln \left(\mathrm{I}_{2}\right)$;

(3) Calculation formula

Ascorbic acid content: $\chi_{\mathrm{C} 6 \mathrm{H} 8 \mathrm{OO} 6}=M_{\mathrm{C} 6 \mathrm{H} 8 \mathrm{O} 6} \times C_{\mathrm{I} 2} \times V$ ${ }_{\mathrm{I} 2} / 2 \times 1000 \times m_{s}$
Mean $\bar{x}: \bar{x}=\frac{1}{n} \sum x_{i}$

Absolute deviation $d: d_{i}=x_{i}-\bar{x}(i=1,2, \cdots)$

Average deviation $\bar{d}: \bar{d}=\frac{\sum_{i=1}^{n}\left|x_{i}-\bar{x}\right|}{n} \quad(i=1,2, \cdots)$

Relative average deviation $d_{r}: d_{r}=\bar{d} / \bar{x}$

Standard deviation of samples $S: S=\sqrt{\frac{\sum_{i=1}^{n}\left(x_{i}-\bar{x}\right)^{2}}{n-1}}$ $(i=1,2, \cdots)$

RSD (relatve standard deviation) $S r: S r=S / \bar{x}$

Standard deviation of sample average $\mathrm{S}_{x}: S_{\bar{x}}=\frac{s}{\sqrt{n}}$

Confidence interval for the mean $\mu$ : $\mu=\bar{x} \pm \frac{t_{a, f} \bullet S}{\sqrt{n}}$

$m_{s}$ is the mass of ascorbic acid, (unit: $\mathrm{g}$ ), $\chi_{\mathrm{C} 6 \mathrm{H} 8 \mathrm{OO} 6}$ is the content of ascorbic acid, $\bar{x}$ is the average value of the measurement, $d$ is the deviation, $\bar{d}$ is the average deviation, $M_{\mathrm{C} 6 \mathrm{H} 8 \mathrm{OO}}$ is the molar mass of ascorbic acid (unit: $\mathrm{g} \cdot \mathrm{mol}^{-1}$ ), $C_{\mathrm{I} 2}$ is the concentration of $\mathrm{I}_{2}$ (unit: $\mathrm{mol} \cdot \mathrm{L}^{-1}$ ), $V_{\mathrm{I} 2}$ is the volume of $\mathrm{I}_{2}$ (unit: $\mathrm{mL}$ ) $S$ is the standard deviation of the sample, and $n$ The number of measurements, $\mu$ is Confidence interval for the mean , $\alpha$ is significance leve, $\alpha=1-P, \alpha=0.05$, $t_{\alpha, \mathrm{f}}$ is a statistic that varies with confidence $P$ and degree of freedom $f, P=1-\alpha=0.95, f=4, t_{\alpha, \mathrm{f}}=$ 2.78 .

\section{Research Content}

(1) First, according to the national standard (GB14754-2010) for the quality control of ascorbic acid in food additives, a mathematical model was established and the programming model was written by VB language;

(2) Analysis and measurement data directly into the computer program, the preparation of a good computer program automatically processing and analysis of measurement data to calculate the results of the analysis, the error, deviation and the average confidence interval; 
(3) The computer automatically calculates the content of ascorbic acid according to the calculation result of the analysis and determination data, and determines the content of various impurities in the sample according to the national standard (GB14754-2010). Meanwhile, the measurement results are statistically analyzed, and the analysis determination of data is accuracy and precision;

(4) All analytical data and data processing results are displayed on the analytical report;

(5) The computer prints automatically the analysis report and save it;

(6) The computer judges automatically the grade of ascorbic acid product (grade);

(7) The computer saves and uploads automatically the batch of ascorbic acid analysis and measurement results and analysis report to the network for users, enterprises and relevant departments to inquire and call at any time on the network;
(8) All data and information uploaded to the network will not be changed [6].

\section{Ascorbic Acid Product Quality Analysis and Determination Application Example}

(1) Quality Analysis and Determinotion of Ascorbic Acid

Double-click the main program to open the ascorbic acid product quality analysis and determination of the main interface of computer management, click the online operation or stand-alone operation to enter the category selection interface, open the project total interface to run the program, click the ascorbic acid analysis determination items. According to national standards, enter ascorbic acid sample after the interface 5, press the button to display the relevant calculation of data, the use of statistical methods to analyze and investigate the data, the results were shown in Figs. 1 and 2 interface.

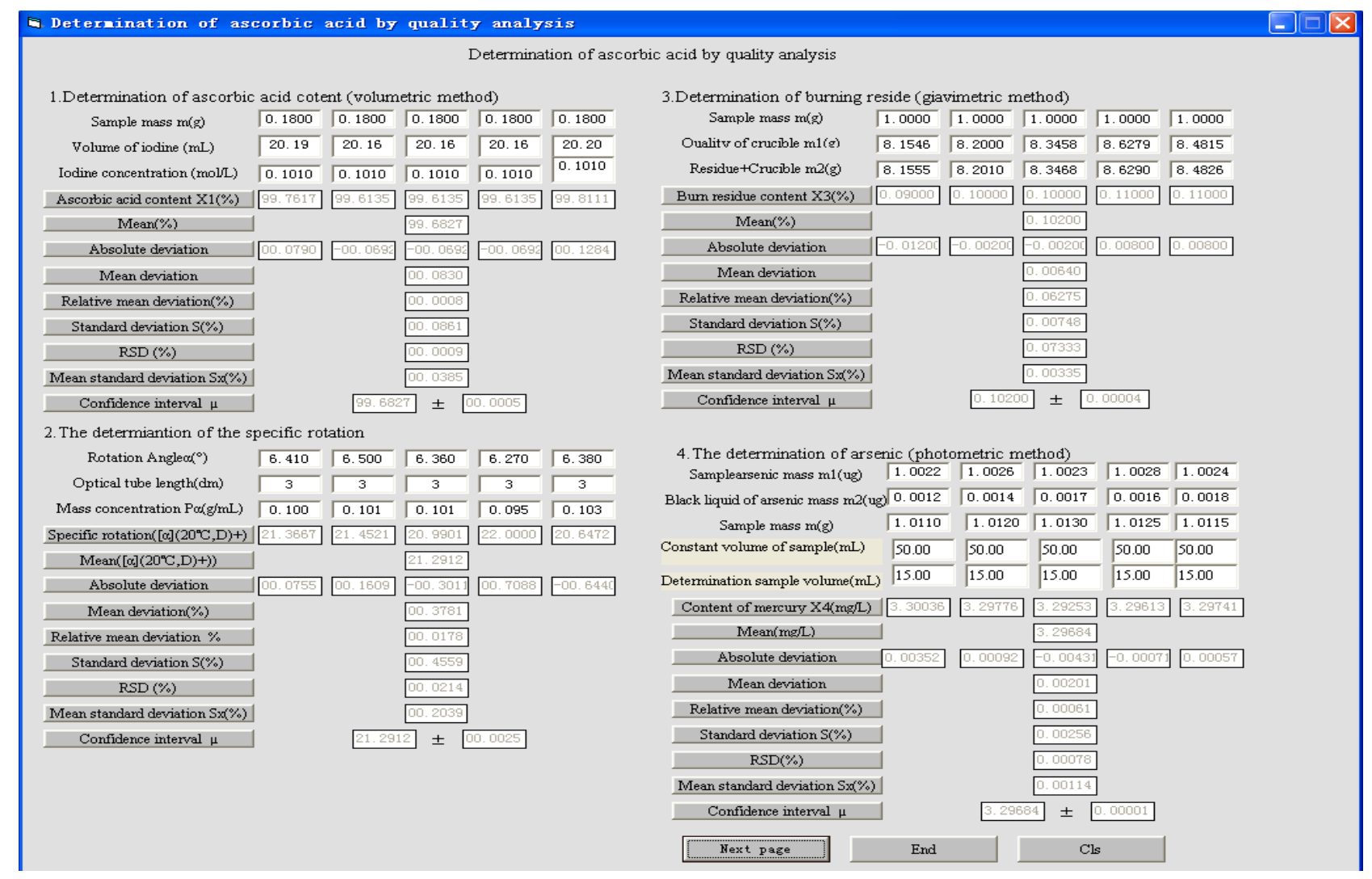

Fig. 1 Ascorbic acid mass percentage and statistical data output interface1. 


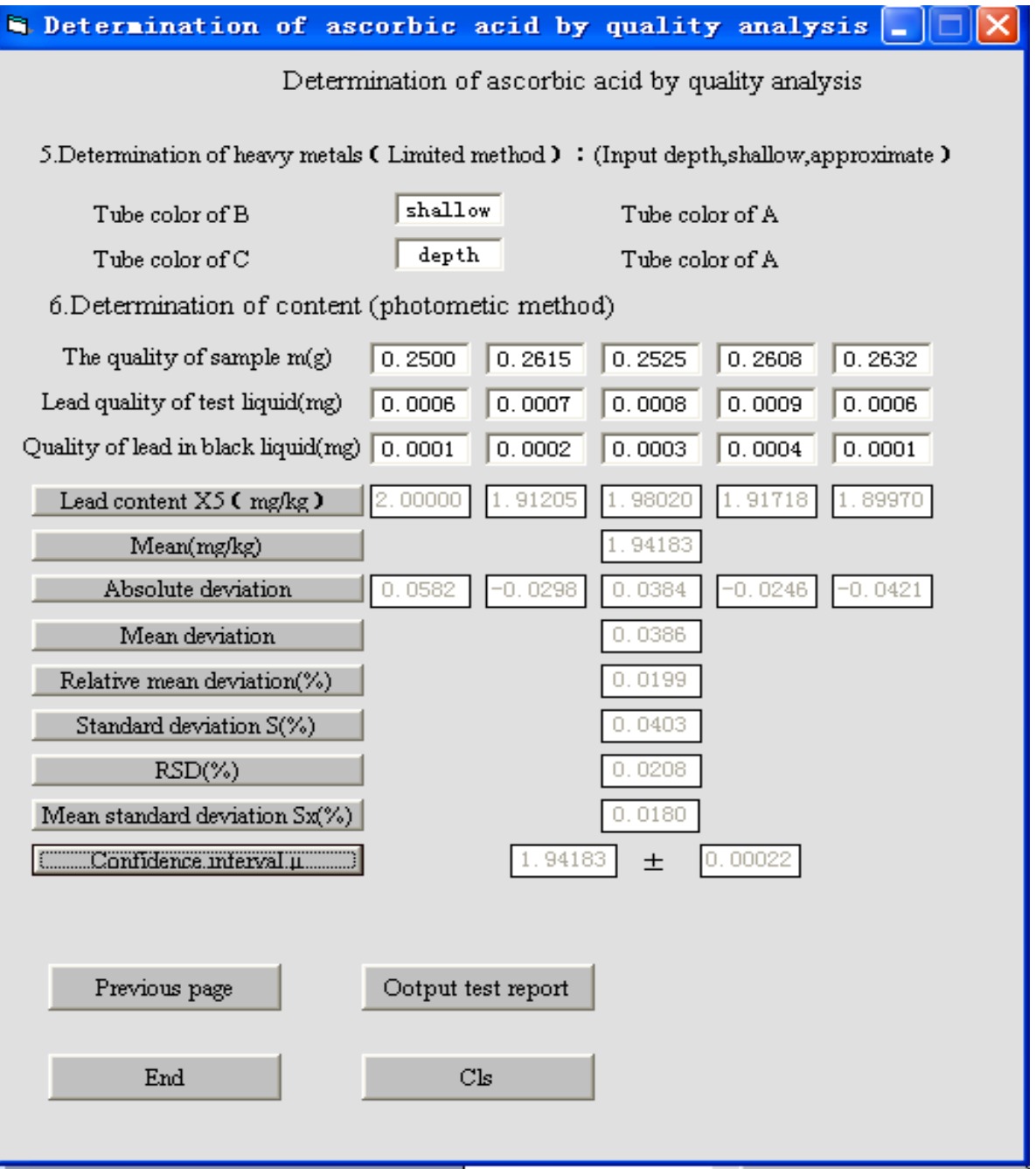

Fig. 2 Ascorbic acid mass percentage and statistical data output interface 2.

Click the "End" button to finish the operation. Click the "Output Determination Result" button to output the analysis report. Click the "Print \& Save" button to print the analysis report.

\section{Conclusion}

The system opens up a new perspective for the application of computer in the quality control of ascorbic acid in food additives, realizing the intelligent management of ascorbic acid quality analysis, improving the management level and the transparency of ascorbic acid quality, avoiding the data no authentic and calculation results inaccurate, eliminating the phenomenon of data changes, reducing the inspection staff to deal with the heavy workload of inspection data, improving work efficiency and the objectivity and fairness of the analysis of test data. The system is practical, targeted, powerful, rapid processing, simple operation, reliable results, which created an advanced, fast, convenient and accurate method for the computer in the food additive ascorbic acid quality analysis test obtained innovative achievements, with a wide range of applicability, promotion and application of a very wide range of prospects.

\section{References}

[1] Zhang, H., Zhang, G., Ye, H., Cui, J., and Qi, J. H. 2015. "Computer Management of Quantitative Analysis Chemistry Experiment." Journal of Chemistry and Chemical Engineering 9 (2): 149-51. 
[2] WuHan University. 2011. Analytical Chemistry Experiment. Beijing: Higher Education Press.

[3] Zhang, H., Zhang, G., Zhang, Q., Cui, J., and Qi, J. H. 2014. "Computer Management of Quantitati Ve Analysia Chemistry Experiment." Journal of Chemistry and Chemical Engineering 8 (2): 176-9.

[4] Zhang, H., Zhang, G., and Ye, H. 2016. "Development and Application of Software in Chemical Product Quality Analysis and Inspection Management System."
Computers and Applied Chemistry 33 (7): 838-42.

[5] Zhang, H., Zhang, G., and Zhang, L. 2015. "Network Management of Food Additives Quality Analysis and Inspection." Journal of Chemistry and Chemical Engineering 9 (7): 468-71.

[6] Zhang, H., Zhang, G., and Zhang, L. 2016. "Intelligent Management Based on Chemical Reagent Quality Analysis." Journal of Chemistry and Chemical Engineerin 10 (7): 336-40. 